AIAA-98-4759

\title{
RESPONSE SURFACE MODEL BUILDING AND MULTIDISCIPLINARY OPTIMIZATION USING D-OPTIMAL DESIGNS
}

\author{
Resit Unal ${ }^{*}$ \\ Engineering Management Department \\ Old Dominion University \\ Norfolk, VA 23529 \\ Roger A. Lepsch ${ }^{\tau}$ and Mark L. McMillin ${ }^{\tau}$ \\ Vehicle Analysis Branch \\ NASA Langley Research Center \\ Hampton VA 23681
}

\begin{abstract}
This paper discusses response surface methods for approximation model building and multidisciplinary design optimization. The response surface methods discussed are central composite designs, Bayesian methods and Doptimal designs. An over-determined D-optimal design is applied to a configuration design and optimization study of a wing-body, launch vehicle. Results suggest that over determined Doptimal designs may provide an efficient approach for approximation model building and for multidisciplinary design optimization.
\end{abstract}

\section{Introduction}

Computerized design and analysis capabilities exist in many key disciplines required for design and analysis of space transportation systems. For a given configuration, system performance characteristics can be determined by the use of these analysis codes. The next step is to determine the settings of design parameters that optimize the system performance characteristics subject to constraints. However, the complex computer programs used in aerospace design are usually stand alone codes run by disciplinary experts. In most cases, they are expensive and/or difficult to integrate and use directly for multidisciplinary design optimization (MDO). An alternative is to construct disciplinary approximation models for the functional relationships between performance characteristics and design parameters. These approximations are referred to as response surface models. The response surface models are then used to integrate the disciplines using mathematical programming methods. In many cases this approach allows efficient system level design analysis, MDO and rapid sensitivity simulations.

\section{Response Surface Model Building Us - ing Central Composite Designs}

A second-order approximation model of the form given below (Equation 1) is commonly used in response surface model building since in many cases it can adequately model the response surface, especially if the region of interest is sufficiently limited.

$$
\begin{aligned}
y= & b_{o}+\sum b_{i} x_{i}+\sum b_{i i} x_{i}{ }^{2} \\
& +\sum \sum b_{i j} x_{i} x_{j}
\end{aligned}
$$

In (1), the $x_{i}$ terms are the input variables that influence the response $y$, and $b_{\mathrm{O}}, \mathrm{b}_{\mathrm{i}}$, and $b_{i j}$ are estimated regression coefficients. The

\footnotetext{
* Associate Professor. This study was funded by NAS1-19858-98.

$\tau$ Aerospace Engineer. Member AIAA.

Copyright (C1998 by the American Institute of Aeronautics and Astronautics Inc. Allrights reserved.
} 
cross terms represent two-parameter interactions, and the square terms represent secondorder non-linearity. Constructing a secondorder model requires that "n" design parameters be studied at least at 3 levels (values) so that the coefficients in the model can be estimated. Therefore $3^{n}$ factorial experiments (design points, functional evaluations or observations) may be necessary. For small values of " $n$ " such as two or three, this approach works well. However, when a large number of design parameters are under study, the number of observations required for a full-factorial design may become excessive. Fortunately, a second-order approximation model can be constructed efficiently by utilizing central composite designs (CCD) from design-of-experiments literature. 1 CCD are first-order $\left(2^{n}\right)$ designs augmented by additional center and "star" points to allow estimation of the coefficients of a second-order model. 1,2

Central composite designs offer an efficient alternative to $3^{n}$ designs for constructing second-order response surface models. A problem involving five parameters for example, requires only $27 \mathrm{CCD}$ experiments to construct a second-order response surface model as opposed to $243\left(3^{5}\right)$ required by a full-factorial study. The authors have utilized CCD for second order approximation model building and MDO in numerous launch vehicle design applications. $^{3,4,5}$ The number of design parameters studied (n) ranged from four to six. In these applications, the fitted second-order model predicted the analysis results with reasonable accuracy within the design region studied.

\section{Response Surface Model Building Us - ing Minimum Point D-Optimal Designs}

CCD enable the efficient construction of a second-order response surface model with significantly less effort than would be required by a full factorial study. However, in some cases, which involve large numbers of design variables, conducting experiments may be timeconsuming and very expensive even with the use of CCD. In such cases, minimum point Doptimal designs may be utilized to generate a design matrix that enables a more efficient construction of a second-order model. 5,6 A design is called "minimum point" when the number of design points is exactly equal to the number of terms in the model to be fitted. ${ }^{6,12,13}$ As a result, minimum point designs require the absolute minimum number of functional evaluations (experiments) to estimate the second-order model coefficients. Compared to minimum point D-optimal designs, CCD are overdetermined designs or there are more experiments than required to estimate the second-order model coefficients.

A statistical measure of goodness of a model obtained by least squares regression analysis is the minimum generalized variance of the estimates of the model coefficients. One way to construct a quadratic model using minimum point designs, leading to minimized variance of the least squares estimates, is to use the D-optimality criterion. Consider the problem of estimating the coefficients of a linear approximation model below by least squares regression analysis; ${ }^{1,5,6}$

$$
y=b_{o}+\sum b_{i} x_{i}
$$

Equation (2) can be expressed in matrix notation as:

$$
\mathrm{Y}=\mathrm{XB}+\mathrm{e}
$$

Where $\mathrm{Y}$ is a vector of observations, $\mathrm{e}$ is the vector of errors, $\mathrm{X}$ is the design matrix and $B$ is a vector of unknown model coefficients $\left(b_{\mathrm{O}}\right.$ and $\left.b_{i}\right)$. The design matrix is a set of combinations of the values of the coded variables, which specifies the settings of the design parameters to be performed during experimentation. B can be estimated by using the least squares method as:

$$
\mathrm{B}=\left(\mathrm{X}^{\prime} \mathrm{X}\right)^{-1} \mathrm{X}^{\prime} \mathrm{Y}
$$

A measure of accuracy of the column of estimators, $\mathrm{B}$, is the variance-covariance matrix which is defined as; $;, 5,6,13$

$$
\mathrm{V}(\mathrm{B})=\sigma^{2}\left(\mathrm{X}^{\prime} \mathrm{X}\right)^{-1}
$$

where $\sigma^{2}$ is the variance of the error. The $V(B)$ matrix is a statistical measure of the goodnessof-fit. Equation 5 indicates that V(B) is a function of $\left(X^{\prime} X\right)^{-1}$ and therefore, one would want to minimize $\left(X^{\prime} X\right)^{-1}$ to improve the quality of the fit. Statisticians have shown that minimizing $\left(X^{\prime} X\right)^{-1}$ is equivalent to maximizing the 
determinant of $X^{\prime} X .{ }^{13,14,15}$ Therefore, generating a design matrix which enables the construction of a good least squares approximation model translates to maximizing the determinant of the $X^{\prime} X$ matrix and experimental designs that maximize $\left|X^{\prime} X\right|$ are referred to as Doptimal designs. $5,6,15$ Here, "D" stands for the determinant of the $X^{\prime} X$ matrix associated with the model. This analysis can easily be extended to the quadratic model given by Equation (1), with the same conclusions for D-optimality. ${ }^{1}$

A number of authors have developed algorithms for obtaining D-optimal designs for specific models using mathematical programming methods. ${ }^{6,13,15}$ There are also numerous software packages available for desk top computers. ${ }^{16}$ A major limitation of a saturated design is the minimum (poor) coverage of the region of interest. ${ }^{17}$ However, D-optimal minimum point designs generally work well in initial screening situations in which it is expected that there will be a few important parameters. ${ }^{15,17}$. As a result, minimum-point $D$ optimal designs may be used in lieu of CCD when there are large numbers of variables under study and experimental design effort is expensive and resources are limited.

\section{Response Surface Model Building Methods for Deterministic Experiments}

For most experimental designs constructed for building response surface models, a dominant issue is the variance of measurements of the response. ${ }^{7}$ However, the output of experiments carried out using computer models, as mostly used in aerospace design, is deterministic. Generally, there is no measurement error or no variability in analysis outputs, given a specific set of inputs. Therefore, experimental designs constructed to minimize variability of measurements and the accompanying statistical methods for model accuracy analysis may not be the best choice for deterministic experiments. 8,9

Reference 8 presents an experimental design approach based on the Bayesian statistics. The approaches for design and analysis of computer experiments using Bayesian statistics are given in references 8 and 9 in some detail. Bayesian approach to experimental design is a growing area of research. However, the appli- cation of Bayesian experimental design methods in practical design analysis and optimization problems seems to have been limited. ${ }^{10}$ Further development appears to be needed before they can be applied to practical design optimization problems.

\section{Using Overdetermined D-Optimal De - signs for Deterministic Experiments}

As noted previously, experimental designs constructed with the objective of minimizing experimental variance may not always be the best choice when approximating deterministic models. Reference 11 conducted a study comparing the performance of experimental design methods for approximation model building for deterministic models in terms of the quality of fit in the region studied and in terms of the number of design points required. The findings suggest that CCD are good designs for problems with five or less design parameters. This is consistent with the authors' experience where good approximations have been obtained using CCD in many applications. $3,4,5$ However, for larger values of $n$, reference 11 recommends the use of augmented minimum point designs that are around $20 \%$ to $50 \%$ overdetermined and, suggests the use of the Doptimality criteria as a heuristic in selecting design points for deterministic experiments.

As a result, the use of $20 \%$ to $50 \%$ overdetermined D-optimal designs for approximation model building appear to be a good choice for response surface model building for deterministic experiments, especially for larger values of $n(n \geq 6)$.

\section{Wing Body Launch Vehicle Configura- tion Study}

An application of approximation model building and MDO using over-determined Doptimal designs is described for a configuration optimization study of a rocket-powered, singlestage-to-orbit launch vehicle. The vehicle is sized to perform a 25,000 lb. payload delivery to the International Space Station from Kennedy Space Center. Near term structures and subsystem technologies are assumed in its design. The vehicle has a wing-body configuration with a slender, round cross-section fuselage and a clipped delta wing. The delta wing 
has elevon control surfaces for aerodynamic roll and pitch control. Small vertical fins, called tip fins, are located at the wing tips for directional control and a body flap extends rearward from the lower base of the fuselage to provide additional pitch control.

In this study, six vehicle design parameters and 15 two-parameter interactions were studied at three values by conducting 45 design experiments as opposed to 729 that would be required by a full-factorial study. The purpose was to determine the best values of the design parameters that satisfy aerodynamic constraints at minimum weight.

To derive the shape of the configuration, response surface methods for MDO were used. At first, a face-centered central composite design (CCD) was utilized. However, the resulting data contained many design points of excessive weight or design points which were very far from being optimal. Using this data, second order approximation equations were generated for weight and for the aerodynamic characteristics of the vehicle at subsonic, supersonic, and hypersonic flight conditions with various control surface settings. The weight equations predicted the design points studied with some crude accuracy. Deviations from the predicted and actual weights (residuals) ranged from $2 \%$ to $9 \%$. The use of transformations (e.g. $\log ($ weight $))$ and the introduction of $\mathrm{cu}-$ bic terms to the model did not improve the prediction accuracy. These results suggested that the use of CCD may not have been the best choice for selecting the experimental design points in this case in the region studied.

In an effort to improve the prediction accuracy, an overdetermined D-optimal design was utilized next. The following steps describe this multidisciplinary optimization and sensitivity study.

\section{$\underline{\text { Identify Design Variables and Feasible Ranges }}$}

Six weight \& sizing and aerodynamics design parameters were varied over a fixed range. The four common parameters included in aerodynamics and weights \& sizing analysis were the fineness ratio (defined as the fuselage length divided by diameter), the wing area, the tip fin area, and the body flap area. The other two parameters were ballast-weight and massratio for weights and sizing analysis and angle of attack and elevon deflection for aerodynamics analysis. The aerodynamics were generated for three different mach numbers, which were Mach 0.3, Mach 2 and Mach 10. As an example, the ranges for the six weights \& sizing parameters are given in Table 1.

Table 1. Weights \& Sizing Parameters and Ranges

\begin{tabular}{lcc}
\hline \hline Parameter & \multicolumn{2}{c}{ Range } \\
\hline Fineness ratio (FR) & 4 & 7 \\
Wing area ratio (WA) & 10 & 20 \\
Tip fin area ratio (TFA) & 0.5 & 3 \\
Body flap area ratio (BFL) & 0 & 1 \\
Ballast weight (BL) & 0 & 0.04 \\
Mass ratio (MR) & 7.75 & 8.25 \\
\hline \hline
\end{tabular}

\section{Construct the Design Matrix}

The next step is to construct an overdetermined D-Optimal design matrix that can enable the construction of a second-order response model for six parameters (Table 2).

Table 2. Six Parameter D-Optimal Design

\begin{tabular}{|c|cccccc|c|}
\hline & FR & WA & TFA & BFL & BL & MR & Weight \\
\hline & & & & & & & \\
1 & -1 & -1 & -1 & -1 & -1 & 1 & \\
2 & -1 & -1 & -1 & -1 & 1 & -1 & \\
3 & -1 & -1 & -1 & 1 & -1 & 0 & \\
4 & -1 & -1 & -1 & 1 & 1 & 1 & \\
5 & -1 & -1 & 0 & 1 & -1 & -1 & \\
6 & -1 & -1 & 1 & -1 & -1 & 1 & \\
. &. &. &. &. &. &. &. \\
. &. &. &. &. &. &. &. \\
. &. &. &. &. &. &. &. \\
. &. &. &. &. &. &. &. \\
41 & 1 & 1 & 0 & 0 & -1 & 1 &. \\
42 & 1 & 1 & 1 & -1 & -1 & -1 & \\
43 & 1 & 1 & 1 & -1 & 1 & 1 & \\
44 & 1 & 1 & 1 & 1 & 0 & -1 & \\
45 & 1 & 1 & 1 & 1 & 1 & 1 & \\
\hline
\end{tabular}

The weights approximation equations from the previous CCD study were used to generate weight predictions for all of the possible $729\left(3^{6}\right)$ combinations of the variables at 
three values. The predictions were not very good, but still useful enough for the purpose of screening out the excessive weight cases. As a result, 91 design points (or parameter combinations) were eliminated due to excessive weight. The remaining 638 design combinations were used as a starting point for generating an overdetermined D-optimal design with 45 design points or experiments (Table 2). ${ }^{16}$

The number of experiments were chosen as 45 for comparison purposes since this many experiments were required by the CCD study. With this new design matrix (Table 2), the six parameters are studied at three levels (values) as represented in coded form by, $-1,0$ and +1 . As an example, a -1 for Fineness ratio corresponds to 4 (lower bound), a 0 corresponds to 5.5 (mid value) and +1 corresponds to 7 (upper bound). These coded values are then transformed into actual parameter values to be used in conducting the analysis. With 45 experiments, this D-optimal design is about $55 \%$ overdetermined since a minimum point Doptimal design would require 29 experiments for constructing the second order model.

\section{Conduct the Matrix Experiments}

In this study, all of the geometry and subsystem packaging of the vehicle were performed using a NASA-developed geometry modeling tool. The Aerodynamic Preliminary Analysis System (APAS) was used to determine vehicle aerodynamics. The weights and sizing analyses were performed using the NASA-developed Configuration Sizing (CONSIZ) weights/sizing package. This process was repeated for the 45 rows of the Doptimal design matrix, each of which corresponds to a vehicle design generating the weights and aerodynamics data.

From the weights analysis 45 data points for, empty weight, landed weight and landing center of gravities were obtained. From the aerodynamics analysis, lift, drag and pitching moment coefficients for the three mach numbers are obtained (Table 3 ).

Construct the Second-Order Response Surface $\underline{\text { Model }}$
Least squares regression analysis is then used to determine the coefficients of the second order approximation model for the weights and the aeroynamics data in terms of the six design parameters. As a result, second order approximation equations for empty weight, landed weight and landing center of gravities (one for payload-in and one for payload-out) were constructed using the weights data. ${ }^{16}$

Table 3. Analysis Results

\begin{tabular}{|c|cccccc|c|c|}
\hline & FR & WA & TFA BFL & BL & MR & Weight & cg \\
\hline & & & & & & & & \\
1 & -1 & -1 & -1 & -1 & -1 & 1 & 268475 & 0.74 \\
2 & -1 & -1 & -1 & -1 & 1 & -1 & 264659 & 0.72 \\
3 & -1 & -1 & -1 & 1 & -1 & 0 & 260610 & 0.75 \\
4 & -1 & -1 & -1 & 1 & 1 & 1 & 322372 & 0.73 \\
5 & -1 & -1 & 0 & 1 & -1 & -1 & 268512 & 0.76 \\
6 & -1 & -1 & 1 & -1 & -1 & 1 & 333966 & 0.78 \\
. &. &. &. &. &. &. &. &. \\
. &. &. &. &. &. &. &. &. \\
. &. &. &. &. &. &. &. &. \\
. &. &. &. &. &. &. &. &. \\
41 & 1 & 1 & 0 & 0 & -1 & 1 & 261486 & 0.76 \\
42 & 1 & 1 & 1 & -1 & -1 & -1 & 235422 & 0.75 \\
43 & 1 & 1 & 1 & -1 & 1 & 1 & 318675 & 0.74 \\
44 & 1 & 1 & 1 & 1 & 0 & -1 & 256535 & 0.75 \\
45 & 1 & 1 & 1 & 1 & 1 & 1 & 326144 & 0.74 \\
\hline
\end{tabular}

Approximation equations for the lift, drag and pitching moment coefficients for each of the three mach numbers are also obtained using the aerodynamics data. These second order approximation models account for individual parameter effects, non linearity (square terms) and interactions (cross terms). The prediction accuracy of the approximation equations in this case was very good (residuals were within $0.50 \%$ to $1.5 \%$ range for the weight equations). These approximation equations can now be used to rapidly determine the effect of varying design parameter values on the weights and aerodynamic performance characteristics and for MDO. A major advantage of this approach is that it enables the integration of disciplines for MDO using mathematical programming methods. Furthermore, sensitivity simulations can be carried out without the need to re-analyze the entire system after each change in parameter values. 
Determine Design Parameter Values that Optimize the Response

In the next step, the approximation equations were transferred into a spreadsheet and a gradient-based non-linear optimizer, contained within the same spreadsheet, is used to determine the settings of design parameter values to minimize vehicle empty weight subject to design constraints. The results are shown in Table 4 .

Table 4. Optimization Process Results

\begin{tabular}{lc}
\hline \hline Parameter & Optimum Value \\
\hline Fineness ratio & 6.9 \\
Wing area ratio & 18.76 \\
Tip fin area ratio & 1.99 \\
Body flap area ratio & 0 \\
Ballast weight & 0.014 \\
Mass ratio & 8.0 \\
\hline Predicted Empty Weight & 249,792 \\
Verified Empty weight & 249,360 \\
\hline \hline
\end{tabular}

Using the optimization results and the empty weight approximation equation, the vehicle empty weight is predicted to be 249,792 pounds. A verification weights \& sizing and aerodynamics analysis using the optimized values was conducted yielding a computed weight of 249,360 pounds. This is very close to the predicted weight. As indicated by this result, the second-order approximation equation constructed using the overdetermined D-optimal design accurately represented the response surface within the region studied for this study.

\section{Conclusions}

In this paper, response surface methods for approximation model building techniques were discussed. A major advantage of using approximation models is that it enables the integration of disciplines for MDO using mathematical programming methods. Furthermore, sensitivity simulations can be carried out without the need to re-analyze the entire system after each change in parameter values.

The approximation techniques discussed were, central composite designs, minimum point designs and overdetermined D-optimal designs for deterministic experiments. A brief summary of each technique was given and an application of model building using overdetermined D-optimal designs for deterministic experiments was presented.

The results suggest that, the use of $20 \%$ to $50 \%$ over-determined D-optimal designs for approximation model building is a good choice for response surface approximation model building.

\section{REFERENCES}

1. R. H. Myers; Response Surface Methodology, Virginia Commonwealth University, Allyn and Bacon Inc., Boston Mass., 1971.

2. Box, G. E. and N. R. Draper, Empirical Model Building and Response Surfaces, John Wiley, New York, NY, 1987.

3. Lepsch, R. A., Jr., D.O. Stanley and R. Unal, "Dual-Fuel Propulsion in Single Stage Advanced Manned Launch System Vehicle," Journal of Spacecraft and Rockets, Volume 32, Number 3, May-June, pp. 417-425, 1995.

4. D. O. Stanley, W. C. Engelund, R. A. Lepsch, M. McMillin, K.E. Wurster, R.W. Powell, A.A. Guinta, and R. Unal, "SSTO Configuration Selection and Vehicle Design," AIAA-93-1053, February 1993.

5. Unal, R., R. A. Lepsch, W. Engelund and D. O. Stanley, Approximation Model Building and MDO Using Response Surface Methods with Applications To Launch Vehicle Design, Proceedings of the 6th Annual AIAA/USAF/ NASA/ISSMO Symposium on Multidisciplinary Analysis and Optimization, September 1996.

6. J. A. Craig, "D-Optimal Design Method: Final Report and User's Manual," USAF Contract F33615-78-C-3011, FZM-6777, General Dynamics, Forth Worth Div.,1978.

7. Sharifzadeh S., J. Koehler, A. Owen and J. Shott, "Using Simulators to Model Transmitted Variability in IC Manufacturing," IEEE Transactions on Semiconductor Manufacturing, Vol. 2, No. 3, August, pp. 82-93, 1989.

8. Sacks, J. W. Welch, T. Mitchell and H. 
Wynn, "Design and Analysis of Computer Experiments," Statistical Science, Vol. 4, Number 4, November, 1989.

9. Owen, A., "Orthogonal Array Designs for Computer Experiments, Department of Statistics, Stanford University,"

http://lib.stat.cmu.edu/designs/owen.small, 1994.

10. Chaloner, K. and I. Verdinelli, "Bayesian Experimental Design: A Review," Department of Statistics, University of Minnesota, Minn., 1995.

11. Carpenter, W. C. "Effect of Design Selection on Response Surface Performance," NASA Contractor Report 4520, June 1993.

12. Lucas, J. M., "Optimum Composite Designs," Technometrics, Vol. 16, No. 4, November 1974.
13. Mitchell, T. J., "An Algorithm for the Construction of D-Optimal Experimental Designs," Technometrics, Vol. 16, No. 2, May 1974.

14. D. C. Montgomery; Design and Analysis of Experiments, John Wiley and Sons, N.J., 1991.

15. M. J. Box and N.R. Draper, "OnMimimum Point Second-Order Designs," Technometrics, Vol. 16, No. 4, November 1974.

16. $\mathrm{JMP}^{\circledR}$ Design User's Guide, SAS Institute Inc, Cary, NC, 1992.

17. D. K. Lin, "Another Look at First-Order Saturated Designs: The P-Efficient Designs,"

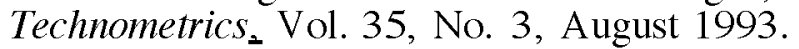

